\title{
Congenital Cervical Aortic Arch Accompanying Aortic Arch Aneurysm: A Case Report
}

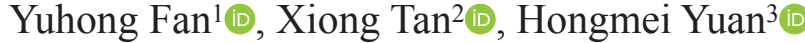 \\ ${ }^{1}$ Department of Ultrasound Affiliated Hospital of North Sichuan Medical College, Sichuan, China \\ ${ }^{2}$ Department of Cardiothoracic Surgery Affiliated Hospital of North Sichuan Medical College, Sichuan, China \\ ${ }^{3}$ Department of Ultrasound Affiliated Hospital of North Sichuan Medical College, Sichuan, China
}

A 29-year-old woman diagnosed with chronic sinusitis was referred to the ENT department of our hospital due to repeated nasal discharge without obvious incentives. Her vital signs were stable, and she had no other clinical symptoms. Two previous cardiac echocardiograms revealed no cardiovascular system diseases. In addition, the patient's medical history, physical examination, and relevant medical test verification did not reveal any other congenital anomalies. Noncontrast computed tomography (CT) revealed an abnormally tortuous aortic arch with local dilation. An emergency bedside ultrasound revealed that the highly tortuous aortic arch was accompanied by local cystic dilation. The patient was finally diagnosed with an aortic arch malformation with an aneurysm (Type D) (Figure 1a, suprasternal notch view; Videos 1 and 2). To confirm the diagnosis, emergency CT angiography was performed, which revealed that the cervical aortic arch had an aortic arch aneurysm and anomalous expansion of both the innominate artery and proximal right subclavian artery (Figure 1b; Video 3) along with atherosclerotic plaques of the descending thoracic aorta. Thereafter, cardiothoracic surgery was performed. During the operation, the aortic arch structure was found to be very tortuous with local expansion and aneurysm formation (Figure 1c). At present, the patient's condition is stable after artificial vessel placement (Figure 1d), and her outcome is under follow-up.
Cervical aortic arch (CAA) is a rare congenital aortic arch malformation. The exact pathogenesis of CAA is still unclear; however, it might be related to the abnormal development of the fourth aortic arch and chromosomal microdeletions during an abnormal embryonic period, which often occur under incidental circumstances. ${ }^{1}$ The most common clinical presentation of CAA is a pulsating neck mass, which can also cause symptoms that indicate airway and esophageal compression. ${ }^{2}$ Haughton et al. classified CAA into types A to E based on the location of the aortic arch and its relationship with the surrounding vessels, with type D being the most common. ${ }^{3}$ CAA exhibits high aortic arch malformation. Moreover, CAA is mostly located between the common carotid artery and subclavian artery and is common in young women. The absolute indications for the surgical treatment of CAA include aneurysm formation, compression symptoms (e.g., dysphagia and dyspnea), and aortic coarctation. ${ }^{2,4}$ The case reported in this study is that of a congenital left carotid aortic arch (Type D) combined with an arch aneurysm, which was surgically removed and replaced by an artificial vessel. To the best of our knowledge, cases of congenital CAA accompanied with an aortic arch aneurysm are very rare; however, this condition is fatal for patients. Through this case report, we hope to draw the attention of medical professionals to this rare and life-threatening condition. 

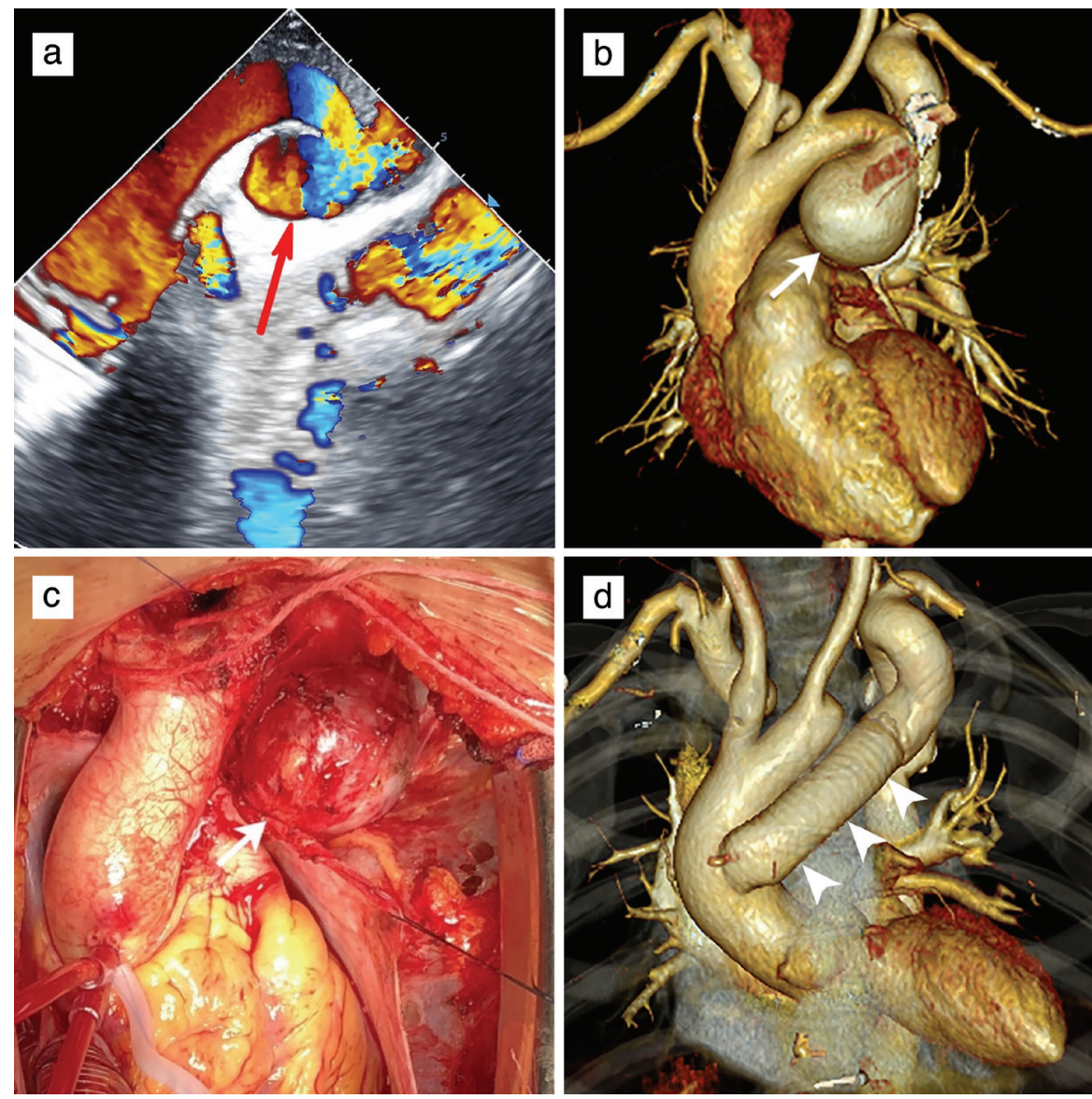

FIG. 1a: Vascular ultrasound of the thoracic aorta showing a tortuous alignment of the aortic arch and a true aneurysm (red arrow) that is visibly inferior to the aortic arch with a red and blue florid blood flow within the aneurysm. b: Preoperative chest CTA showing aortic arch malformation, a cervical aortic arch with an aneurysm visible under the arch (white arrow), and anomalous expansion of the innominate artery with a slightly tortuous course. c: Intraoperative picture showing the cervical aortic arch accompanying aortic arch aneurysm (white arrow). d: Post-operative chest CTA showing the surgically removed aneurysm, wherein the proximal end of the incision was sutured and an artificial vessel (arrowheads) was used to connect the distal end of the incision to the ascending aorta.

Patient Consent for Publication: Written informed consent was obtained from the patient for the publication of this article, including the accompanying data.

Authorship Contributions: Concept- X.T.; Critical Review: H.Y.; Writing - Y.F.

Conflict of Interest: No conflict of interest was declared by the authors.

Funding: This work was financially sponsored by the Sichuan Science and technology project (No. 2019YJ0710).

Acknowledgments: We are grateful to Prof. Tianwu Chen, Prof. Ping Lv and the members of the ultrasound department for their generous help.

\section{REFERENCES}

1. Beller JP, Kron IL. Commentary: Cervical aortic arch repair-an overarching success. J Thorac Cardiovasc Surg. 2020;159:2214-2215. [CrossRef]

2. Steele L, Silver B, Beegun I, Saleh H. Cervical aortic arch: an unusual cause of a pulsatile neck mass. BMJ Case Rep. 2018;2018:bcr-2018-224515. [CrossRef]

3. Haughton VM, Fellows KE, Rosenbaum AE. The cervical aortic arches. Radiology. 1975;114:675-681. [CrossRef]

4. Makani S, Mitchell J, Metton O, Di Filippo S, Henaine R, Ninet J. Surgical repair of a pseudocoarctation with cervical aortic arch complicated by multiple aneurysms of the aorta: a case report. Pan Afr Med J. 2017;26:236. [CrossRef] 